\title{
PERCEPCIÓN DE LA REALIDAD Y PREHISTORIA. RELACIÓN ENTRE LA CONSTRUCCIÓN DE LA IDENTIDAD Y LA COMPLEJIDAD SOCIO-ECONÓMICA EN LOS GRUPOS HUMANOS
}

\author{
PERCEPTION OF REALITY AND PREHISTORY. \\ RELATION BETWEEN CONSTRUCTION \\ OF IDENTITY AND SOCIO-ECONOMIC COMPLEXITY \\ IN HUMAN GROUPS
}

ALMUDENA HERNANDO

$(*)$

\section{RESUMEN}

Ante el reciente auge del interés por los aspectos cognitivos de las sociedades de la Prehistoria y del uso que de la hermenéutica fenomenológica se está haciendo para este fin, propongo en este trabajo varias líneas de reflexión: a) analizar las razones de ese interés en los aspectos "mentales" del pasado. b) Comprender las bases de interpretación y posibilidades de aplicación de la hermenéutica fenomenológica, inapropiadas a mi juicio para la interpretación de la Prehistoria; y c) justificar la necesidad de comprender otros órdenes de racionalidad para poder comenzar a plantear el desarrollo cultural de las sociedades de la Prehistoria.

\begin{abstract}
The cognitive aspects of past societies are receiving progresive attention in Archaeology. Phenomenological hermeneutics are being used as the philosophical basis for the study of history. I will try to show: a) why the Social Sciences are interested in those mental aspects of other societies. b) Why phenomenological hermeneutics are not adequate to analyze them; and c) the existence of different orders of rationality among cultures and the need to understand them if we want to analyze past behaviours.
\end{abstract}

(*) Dpto. de Prehistoria. Facultad de Geografía e Historia. Universidad Complutense. Madrid. Correo electrónico: hernando@eucmax.sim.ucm.es.

El artículo fue remitido en su última versión el 8-IX-99.
Palabras clave: Arqueología Cognitiva. Hermenéutica. Estructuralismo. Tiempo. Espacio.

Key words: Cognitive Archaeology. Hermeneutics. Estructuralism. Time. Space.

\section{INTRODUCCIÓN}

Cualquier estudio historiográfico que contemple las circunstancias socio-económicas en que se han desarrollado nuestras disciplinas científicas demuestra que el contenido de nuestros discursos de conocimiento está determinado por los intereses, conflictos, preocupaciones y sensibilidad general de cada época. La convicción se incrementa cuando la referencia es a las Ciencias Sociales, donde la trasposición de preocupaciones e intereses puede llegar a ser casi directa, alcanzando lo que los psicológos denominarían su "proyección" desde el presente. En efecto, una mirada a los objetivos de nuestros estudios en Prehistoria nos enseña, por ejemplo, que dependiendo de las fases históricas por las que ha atravesado Europa, nuestra búsqueda en el más remoto pasado se ha centrado consecutivamente en:

1. sus objetos materiales, cuando se iniciaba el mercantilismo capitalista en el ámbito económico y los nacionalismos en el político, y el desarrollo de los hábitos de consumo exigía un incremento de la atención a los objetos y una especialización 
del deseo por los generados dentro de nuestra sociedad.

Esta etapa, al ser la primera, revistió además un fondo teórico evolucionista. La Prehistoria nació vinculada a las Ciencias Naturales en un esfuerzo común de todas las nuevas disciplinas por renovar la construcción social de la realidad esencial al desarrollo del capitalismo. Para ello necesitaban sustituir el espacio y el estatismo que caracterizan al Mito por el tiempo y el cambio que definen a la Ciencia, y ello exigía, a su vez, probar que la historia de cualquier fenómeno - geológico, biológico o cultural-, se caracterizaba por una sucesión de transformaciones hacia la progresiva complejidad. Por ello, cuando Lubbock sintetizó su primera visión de la Prehistoria en su Prehistoric Times de 1865, estableció estadios sucesivos definidos cada uno de ellos por una innovación tecnológica que, en la filosofía ilustrada, demostraría la tendencia innata de la humanidad a mejorar sus condiciones de vida a través de la razón. Los objetos materiales estaban revestidos, así, de un significado muy profundo, pues se convertían en las evidencias manifiestas de dicha tendencia.

A su vez, el evolucionismo (Elías, 1993: 22-31) caracterizaba un momento en el que el progreso industrial ponía de manifiesto dos factores: la posibilidad de claras mejoras en las condiciones materiales de vida por un lado, y la distribución irregular de dichas posibilidades, que sólo afectaban a unos cuantos, por otra. Por ello, es lógico que la preocupación básica de los pensadores de la época reflejara una idealización del futuro, en el que la generalización de ese progreso material podría ser un hecho. A medida que la nueva sociedad capitalista iba desarrollándose, la preocupación por los objetos iba siendo sustituída, en consecuencia, por la preocupación por las relaciones sociales. Ya no hacía falta demostrar la transformación que caracterizaba a las sociedades, pues dicha variable había pasado a formar parte de la "mentalidad" de la sociedad europea, de su percepción de la realidad. Y así, la Prehistoria, al igual que todas las Ciencias Sociales, entrará en una nueva etapa, definida ahora por su interés en:

2. las relaciones sociales. Podría decirse que esta etapa tiene dos fases, aunque se define como paradigma en Prehistoria sólo en la segunda de ellas. La primera manifiesta ese deseo de transformación, se hace cargo de la palpable y omnipresente injusticia social que perpetúa el naciente capitalismo. El deseo de cambio, de generalización del nivel de bienestar, de una ausencia de explotación entre las clases y la evidencia del conflicto social interno constituía así tanto el estado de la sociedad del momento, como los ejes principales sobre los que estructurar las teorías con que analizar el pasado. El materialismo dialéctico, formulado desde mitad de siglo XIX, pero aplicado a las Ciencias Sociales hasta incluso nuestros días, representa esas preocupaciones. Gordon Childe ayudó a sintetizar el particularismo previo de los objetos con las corrientes marxistas tras el impacto de su visita a la URSS en 1935 (Trigger, 1992: 239-240), y desde entonces han sido varios los representantes de esta tendencia en Prehistoria y Arqueología (cfr. Mc Guire, 1992).

Ahora bien, los arqueólogos, integrantes de la clase intelectual y funcionarial de la sociedad occidental, no han solido ser representantes de las clases a las que no pertenecían, las más desfavorecidas. Por ello, cuando el énfasis en los objetos empezó a resultar obsoleto porque se hacía evidente su incapacidad para la explicación histórica, la mayor parte de ellos, conscientes de la transformación de las estructuras y relaciones sociales que acompañaban el desarrollo del nuevo orden económico -o que permitían que ése se desarrollara, pues ambos forman parte del mismo proceso de transformacionescomenzó a centrar la atención en el orden social, pero desde su propia posición de "favorecidos".

Así, puede hacerse referencia a una segunda fase dentro de los estudios prehistóricos en que las relaciones sociales han constituído el núcleo fundamental de atención, pero donde, a diferencia de la anterior, ya no se idealiza el futuro, sino que el mantenimiento de las condiciones de bienestar alcanzadas en el presente y del privilegio del que se participa dentro de esa distribución, constituyen el ideal (Elías, 1993: 29). Esta percepción de la propia situación en la sociedad, y la suposición de que se está hablando de presupuestos objetivos cuando sólo se están proyectando las condiciones subjetivas de las que gozan los intelectuales europeos, permitió la aparición del funcionalismo como corriente dominante en los años 60 .

En realidad, ambas corrientes teóricas -materialismo dialéctico y funcionalismo- constituyen la concreción en Ciencias Sociales del paradigma evolucionista multilineal, que se había venido gestando en Prehistoria y Arqueología desde los años 30 de nuestro siglo. En Europa, tres influencias distintas se dieron cita para conseguir relegar el historicismo de los objetos a un segundo plano, imponiendo

T. P., 56, n. ${ }^{\circ} 2,1999$ 
el intento de formulación de leyes generales sobre el comportamiento social: 1) la tradición ecológica escandinava, desarrollada ya desde mitad del siglo XIX, cuandoWorsaae defendía la necesidad de estudiar los hallazgos arqueológicos en relación a su entorno paleoambiental (Trigger, 1992: 233); 2) la Arqueología Soviética, que desde los años 20 intentaba un análisis materialista de la condición humana, pasada y presente (Trigger, 1992: 205-209); y 3) el desarrollo del funcionalismo en los estudios etnológicos, constituyendo la corriente de la Antropología Social. En el Reino Unido, se produjo una reacción contra el historicismo y difusionismo previo de Elliot Smith a través de la adopción del enfoque estructural-funcionalista de Malinowski y Radcliffe-Brown, cuyas dos obras más importantes fueron publicadas en 1922. Ambos insistían en la necesidad de comprender el comportamiento humano en relación a los sistemas sociales donde se insertaban, concibiendo éstos como un cojunto de elementos funcionalmente interdependientes. Este enfoque se basaba, a su vez, en los estudios de E. Durkheim (1858-1917), quien al igual que Marx, concebía las sociedades como conjuntos interdependientes de elementos (Trigger, 1992: 231).

Podríamos decir que el materialismo histórico de V. Gordon Childe fue el resultado concreto de la unión de las influencias 2 y 3 -Arqueología Soviética y Funcionalismo-, mientras que el funcionalismo conservador de Grahame Clark lo fue de la síntesis de las influencias 1 y 3 -tradición ecológica escandinava y funcionalismo- (Trigger, 1992: 247-8).

Por su parte, el evolucionismo multilineal en Estados Unidos tuvo sus principales representantes en A. Leslie White y en J. Steward, en los años 40 y 50 . Ya en los años 20, Leslie White se había interesado vivamente por la Teoría Social, visitando incluso la Unión Soviética en 1929, y en los años 30 , J. Steward había empezado a formular su teoría de la Ecología Cultural (McGuire, 1992: 70). De hecho, las dos décadas que siguieron a la Segunda Guerra Mundial fueron una época de prosperidad económica sin precedentes en los Estados Unidos, que al tiempo contemplaba una hegemonía política incuestionable. La autoconfianza que esto generó explica, en parte, el auge que allí habrían de tomar los modelos funcionalistas-materialistas, y la creencia de que el progreso tecnológico era la clave de la superación histórica (Trigger, 1992: 271).

En efecto, a partir de los años 60, de manera clara en Estados Unidos -con la Nueva Arqueología, li- derada por L. Binford-, y un poco más tarde en Europa -que tenía que recuperarse de las dos guerras mundiales sufridas en su suelo-, la sociedad del bienestar era ya un hecho para una parte significativa de la sociedad. La clase media se iba haciendo dominante y aunque semejante grado de bienestar se conseguía a través de la explotación de un sector desfavorecido de ella, y sobre todo, del resto del mundo no-europeo, la mayor parte de la población no tenía ya esa urgencia en transformar sus propias condiciones de vida. Además, ahora, por primera vez, las dos clases industriales se empezaban a integrar en el entramado estatal, pues el el acuerdo de los representantes del proletariado industrial comenzaba a resultar esencial para gobernar los estados de la sociedad occidental (Elías, 1993: 27).

Por todo ello, el funcionalismo comenzó a proyectar al pasado una idea de la sociedad como un todo armónico que tiende al equilibrio - no al cambio-, y en el que todas las partes cumplen una función positiva en el conjunto. Si nos detuviéramos sólo un momento a reconocer, como sugiere Elías (1993: 29) hasta qué punto estos modelos de "sistema social" son inadecuados como instrumentos teóricos para la investigación científica de sociedades caracterizadas por la desigualdad, como las sociedades esclavistas, las feudales o las estamentales -por no salir de nuestra propia trayectoria histórica-, no nos quedaría otro remedio que reconocer que los modelos teóricos utilizados para analizar el pasado están, en realidad, centrados en el presente.

Ahora bien, a medida que la sociedad del siglo XX ha ido avanzando y la generalización del bienestar afectando a un mayor porcentaje de población, se ha ido comprobando también que el bienestar material no implica el psicológico. De hecho, el aumento de las disfunciones de este tipo es correlativa al incremento de la renta per cápita de los prósperos países europeos y norteamericanos. Los índices de depresión y suicidio juvenil están conociendo cotas inimaginables hasta hace muy poco tiempo, lo que empieza a hacer que centremos la atención en los mecanismos de la mente como contexto problématico, en lugar del económico o del social -resuelto para esa parte de la población que puede dedicar sus energías al estudio de la Prehistoria, y cuyos hijos, alumnos o conocidos son los que se deprimen y suicidan-. De hecho, ya las propias tendencias marxistas, a través de la Escuela de Franckfurt, habían generado desde hacía tiempo un interés por los estudios sobre la "falsa conciencia" o modo particular y subjetivo de percibir la realidad 
desde distintas posiciones sociales, para enmascarar sus problemáticas actitudes dentro de ellas. Por su parte, la Arqueología británica -sin que sea casual, a mi juicio, el carácter protestante y, en consecuencia, individualista de la sociedad anglosajona-, comenzó a desarrollar posiciones relativistas y subjetivistas a través de la llamada corriente postprocesual, personalizada en la figura de Ian Hodder desde los años 80. Pero es fundamentalmente a partir de la década de los 90, en que la trayectoria social del mundo moderno-occidental implica una generalización del individualismo a cualquier contexto europeo y norteamericano-lo que va asociado a una transformación de la relación subjetiva con la realidad-, cuando los estudios de Prehistoria están empezando a centrar la atención en:

3. Las cuestiones cognitivas o el funcionamiento de la subjetividad. Existe además otro tipo de factores que inciden en el estudio de las "ideas" y de la subjetividad humana y en su desarrollo en la Prehistoria: el individualismo extremo a que va conduciendo la sociedad post-moderna implica un paralelo abandono de la lucha social. El individuo se va convirtiendo en referencia básica desde la que percibir la realidad, y por tanto, desde la que juzgarla, y a medida que su identidad es menos colectiva, la formación de "bloques" de lucha se hace más difícil. A partir de ahora la sensibilidad y preocupación por los desfavorecidos se manifiesta de forma progresiva en la participación solidaria, pero no política, en la actividad que las ONGs van desarrollando para rellenar los vacíos de atención social del estado del bienestar. Resulta difícil imaginar un mejor acuerdo entre necesidades individuales y conveniencias políticas, una manera más ajustada de atender la desigualdad sin entrar en conflicto con quien la provoca o, al menos, la mantiene. $Y$ es que, ahora, el locus del conflicto ha pasado de lo social a lo estrictamente individual, del mundo extra-psíquico al intra-psíquico.

Por otra parte, dado el individualismo extremo de la sociedad norteamericana, y dado que en este momento hay más énfasis en el mantenimiento de la situación presente que en la transformación para el futuro que venía asociada a la idea de progreso que guiaba a sus "pioneros", su población no distingue claramente entre los rasgos liberales y los conservadores, que ni siquiera se consideran contrapuestos (Elías, 1993: 27). Con la misma idealización del presente, pero sustituyendo el valor explicativo que en el funcionalismo tenía el grupo social por el papel del individuo en coherente corre- lación con la desmembración individualista de la sociedad post-moderna, estamos viviendo un auge de los modelos "interpretativos", hermenéuticos, del pasado prehistórico, cuyo grado de compromiso social parece, en todo caso, reducido, y cuyo riesgo de solipsismo y diletantismo es grande.

Como era de esperar, la bandera del nuevo paradigma está en manos anglosajonas, cuyo individualismo protestante sigue llevando la delantera a la más colectivista identidad católica del centro y sur de Europa. Por ello, la muy prolífica producción británica y americana empieza a llenarse de títulos que hacen alusión al "significado" o la "interpretación", frente a los esfuerzos por calar en las condiciones sociales del pasado que definieron la disciplina arqueológica de otras décadas (Hodder et alii, 1995; Shanks, 1992; Barret, 1994; Mithen, 1996; Bradley, 1998; Renfrew y Zubrow, 1994, por ejemplo).

La llamada "Arqueología Interpretativa", o "Cognitiva", representa el nuevo paradigma, como correlato a la "Antropología Interpretativa" que, en palabras de E. Gellner (1995: 48), constituye "el desarrollo teórico más conspicuo y visible de la Antropología Social de los últimos años". Incluso los funcionalistas hacen esfuerzos por incorporarse a la nueva corriente (Renfrew, 1993 y 1994), ante la evidencia de que el análisis de otras culturas - tanto en el tiempo como en el espacio- se hace desde las pautas de percepción de la realidad que caracterizan a la del investigador, lo que introduce el reconocimiento de factores subjetivos en la investigación que hasta hace poco no se tenían en cuenta.

El creciente individualismo de nuestra sociedad va transformando el tipo de relaciones que sostenemos tanto con nosotros mismos, como con el resto de la sociedad, o con el conjunto de la realidad en la que vivimos. Cada vez nos sentimos más aislados, nuestro modo de identidad se basa más en la diferencia que nos distingue de los demás que en la similiaridad que nos une al resto del colectivo al que pertenecemos. Y por eso, cada vez somos más conscientes de que existen modos distintos de percibir la realidad, de entenderla, de manejarla. En consecuencia, se nos empieza a abrir la posibilidad de aplicar nuestra propia experiencia a otras culturas, para aceptar que quizás los "otros" no entendieron -o no entienden- las cosas del mismo modo, no tuvieron los mismos deseos y no persiguieron los mismos objetivos. Vamos teniendo cada vez más claro que la relación material con la realidad -único aspecto tenido hasta ahora en cuenta por la Pre-

T. P., 56, n. ${ }^{\circ} 2,1999$ 
historia- depende de la percepción que de ella tengamos en una relación de mutua dependencia, como ya venían reclamando desde hace tiempo los estructuralistas (Criado, 1993: 41). Por tanto, si queremos comprender otras culturas, debe ser necesario investigar cómo percibían ellas su propia realidad.

Como podrá imaginarse, esta tendencia no es particular de la Prehistoria, sino que se manifiesta en los últimos años en todas las Ciencias Sociales, en tanto que la Ciencia fue el modo de relación con la realidad que sustituyó al Mito a partir de la Modernidad (Hernando, 1997). Es decir, la Ciencia constituye uno de los mecanismos esenciales de orientación e identidad en la sociedad industrial y post-industrial, por lo que será expresión de las preocupaciones y necesidades de quienes la formulan. De ahí que todas las disciplinas que intentan dar unas pautas de localización existencial a la sociedad actual sobre quiénes somos en realidad -a través de la investigación de nosotros mismos o de lo "otro"reflejen el mismo interés por analizar la percepción. Buena prueba de ello es, por ejemplo, la muy reciente disciplina de la "Psicología Cultural" que, nacida en Estados Unidos en esta última década, se propone investigar los mecanismos mentales de otras culturas (Shweder, 1991; Stigler et alii, 1990) de forma más introspectiva y personal aún que lo que llega a hacer la llamada "Antropología de Uno Mismo" (Anthropology of Self) (Erchark, 1992).

Es precisamente en el grado de "subjetivismo" y en los métodos de análisis de "conciencias pasadas" donde me gustaría incidir en este artículo, porque creo que estamos viviendo un momento de cierta pérdida de referentes teóricos en el estudio de la Prehistoria, y que ni los procesuales por un lado, ni los post-procesuales, que han asumido sin crítica lo que Gellner denominó la "vuelta de tuerca hermenéutica" (hermeneutic twist) por otro, consiguen desarrollar un marco de interpretación válido para acceder con cierto rigor al problema de la percepción de la realidad en el pasado.Ambas resultan epistemológica y metodológicamente incapaces de "escapar" a la "mente" del investigador, y además, los post-procesuales, al basarse en la hermenéutica fenomenológica e intentar comprender así "intuitivamente" el significado de los hechos del pasado, abandonan cualquier análisis del contexto socio-económico donde se generaron, bloqueando las posibilidades de conocimiento de las condiciones reales en las que se desarrolló el proceso a investigar. El principal obstáculo que, a mi juicio, presentan los estudios cognitivos o interpretativos más recientes es que no tienen en cuenta que la cultura es un conjunto integrado de rasgos, un fenómeno complejo y amplio en el que las condiciones materiales y las cualidades subjetivas de percepción de esas condiciones exigen coherencia entre sí, se transforman correlativamente y están mutuamente determinadas. En consecuencia, sus posibilidades de comprensión de las culturas del pasado es tan limitada como la de su polo opuesto, los materialistas positivistas que, en recíproca actitud, niegan el papel fundamental de la percepción de la realidad en la valoración de una cultura.

Quiero detenerme en esta cuestión en las páginas que siguen, y utilizar el espacio del que dispongo para plantear lo que, a mi juicio, constituyen las premisas básicas sobre las que asentar futuros esfuerzos. Como podrá haber empezando a deducirse, considero que el Estructuralismo constituye la única vía de escape a las limitaciones de laArqueología procesual y post-procesual en lo que al acercamiento a algunos de los aspectos cognitivos del pasado se refiere.

\section{ESTUDIOS COGNITIVOS EN PREHISTORIA}

Resulta difícil comprender el énfasis en los estudios de la cognición en Prehistoria sin tener en cuenta que forman parte de la lógica correspondiente al estado de cultura en el que nos encontramos, la llamada Post-modernidad. Ahora bien, dentro de ella, existen corrientes diferentes que, aunque parecen tener un objetivo común, en realidad parten de posiciones filosóficas muy distintas. Digamos que algunas de ellas siguen consistiendo esencialmente en propuestas modernas, mientras que otras son ya, realmente, post-modernas. Intentaré hacer un esquema de conjunto que sirva para esclarecer en la medida de lo posible lo que entendemos por propuestas cognitivas o Arqueología Cognitiva.

Como luego tendremos también ocasión de ver, la Modernidad se definió esencialmente por dos rasgos culturales: el desarrollo de la individualidad y la prioridad del conocimiento científico. En efecto, la Modernidad podría caracterizarse por un desarrollo extremo de la individualidad, pues presenta el máximo grado de esferas de relación, actuación social, división de funciones y especialización del trabajo de todas las formaciones sociales que han existido en la Historia. Eso hace que las personas 
que la representan tengan que desarrollar un alto control de las emociones que les permita actuar con distintos niveles de implicación y distancia emocional en cada uno de esos niveles y esferas, lo cual produce, a su vez, la conciencia de un conjunto de emociones reprimidas o controladas y esto la percepción de un "yo" particular, personal e intransferible, que nos distingue en lo más profundo del resto de los seres que tenemos alrededor. Es decir, a partir de un cierto momento, la sociedad empezó a estar formada por "individuos" que se creían (nos creemos) aislados de la realidad que les rodeaba, o lo que es igual, que creían que existía una distancia entre ellos y el resto de la realidad. Y esta distancia les permitía observar con perspectiva, sin implicación emocional esa realidad. La Naturaleza no-humana, considerada como algo distinto y distanciado de la Naturaleza humana comenzará así, a poder ser explicada en sus propios términos, para lo que se generarán los modos científicos de conocimiento. Por eso la Ciencia se generaliza en la Modernidad, si bien su inicio coincide con todos los episodios históricos en los que ha habido un desarrollo de la individualidad porque la división de funciones dentro de la sociedad permitía, al menos a determinados individuos, adoptar posiciones, y por tanto, percibirlas, como algo diferente del resto de su comunidad social.

Digamos así que la Modernidad está basada en dos premisas esenciales, pero contradictorias: la Subjetividad o percepción de la existencia de esa individualidad, y la Razón Universal, o creencia en que los fenómenos de la Naturaleza pueden ser explicados conforme a modelos propios de funcionamiento. Durante la Modernidad, la segunda premisa tenía mucho más peso que la primera, pues el nuevo Sujeto estaba conformándose aún y, aunque ya había conciencia de diferenciación con el resto de los miembros del grupo social, todavía existía una sociedad donde las relaciones -familiares, de clase, sociales-eran un factor muy importante de identidad. De esta forma, podemos decir que el fenómeno más visible durante esa etapa fue el desarrollo vertiginoso, la irrupción explosiva, la intensa y sorprendente generalización de la Ciencia como nuevo modo de relación con la realidad, de comprender cualquier aspecto de ella, en el que se incluía el propio sujeto de la Modernidad que intentaba ser explicado y contextualizado a través de las recién creadas Ciencias Sociales.

Ahora bien, Subjetividad y Razón Universal son dos términos contradictorios. O bien se cree que la realidad puede explicarse conforme a dinámicas objetivas, buscando por ello las leyes que rigen los fenómenos que la caracterizan sin que el observador afecte o modifique su funcionamiento (y esto es el positivismo), o bien se cree que el observador determina, en el propio acto de la observación, el carácter de lo observado, pues lo selecciona e interpreta conforme a sus condicionantes subjetivos y personales. Así que, a medida que esta sociedad capitalista nuestra fue ahondando en su propia lógica de desmembración y fragmentación social, de división de funciones y especialización del trabajo, que obliga a los individuos a situarse cada vez más en posiciones y roles cambiantes -lo que va incluyendo como no sucedía al comienzo de la Modernidad a las mujeres, por ejemplo- para sobrevivir material y socialmente, la conciencia de la diferenciación con respecto a los demás aumenta, y con ello, el primer polo del par: el peso de la Subjetividad en nuestra percepción de la realidad. Es decir, poco a poco, al tener progresivo peso un modo de identidad individualizado, vamos teniendo una mayor conciencia de que nuestra percepción del mundo depende, en cierta medida, de nuestras condiciones particulares de vida. De esta forma, aunque seguimos manteniendo una mayor independencia en nuestra visión de la Naturaleza no-humana, y por tanto, confiando aún de forma generalizada en la Razón Universal en las llamadas Ciencias Físicas o Naturales, comienza a quebrarse la seguridad que antes teníamos en la objetividad con que analizamos los fenómenos de la Naturaleza humana, y con ello, los modelos positivos de las Ciencias Sociales. Nos empieza a parecer que, quizás, los modelos teóricos que rigen la Historia o la Antropología o la Sociología están mediatizados por nuestra visión del presente, y que, por tanto, en lugar de explicar los fenómenos humanos, en realidad lo que hacemos es interpretarlos siempre.

La Post-modernidad podría definirse como la crisis de la Modernidad provocada por la ruptura que implica el triunfo de la Subjetividad frente al dominio de la Razón Universal que caracterizó a la Modernidad. De esta forma, podría decirse esquemáticamente, que en el binomio Subjetividad vs. Razón Universal, las tendencias que aún dan prioridad al segundo polo pueden definirse como modernas, mientras que las que se lo dan al primero son post-modernas, aunque ambas se hacen eco, irremisiblemente, de la creciente conciencia de la subjetividad que el imparable desarrollo de la individualidad de nuestra sociedad implica, y de ahí 
que ambas generen y se interesen por cuestiones cognitivas.

Por otra parte, las sociedades humanas pueden analizarse desde el punto de vista del individuo o de la sociedad. Tanto con el lenguaje como con el pensamiento parecemos tratar a ambos como si fueran dos manifestaciones con existencia separada, una de ellas real -los individuos-y otra irreal-la sociedad-(Elías, 1993: 30). Sin embargo, cuando hablamos de "individuos" nos estamos refiriendo a un particular modo de concebir a cada integrante de un grupo humano, y cuando lo hacemos de "sociedad", estamos aludiendo al conjunto de interrelaciones que ésos establecen entre sí. Así pues, no son más que dos caras de una misma moneda a través de la cual encarar el análisis de la Naturaleza humana y de todos los fenómenos que la caracterizan.

De esta forma, podría decir que las posiciones teóricas desarrolladas para ello pueden clasificarse teniendo en cuenta la combinación que establecen entre los cuatro factores siguientes:

- Por un lado, individuo y sociedad.

- Por otro, subjetividad y razón universal.

Desde un énfasis moderno, caracterizado por la prioridad dada a la razón universal:

- el estudio del individuo ha dado lugar a las posiciones historicistas;

- el estudio de la sociedad ha dado lugar a las posiciones materialistas y funcionalistas.

Desde un énfasis post-moderno, caracterizado por la prioridad dada a la subjetividad en la relación con la realidad,

- el estudio del individuo ha dado lugar a las posiciones hermenéuticas;

- el estudio de la sociedad ha dado lugar a la Escuela de Franckfurt.

Creo que habría que reservar un lugar aparte en este esquema para el Estructuralismo, pues constituye una posición intermedia entre ambas. Es el intento de estudiar objetivamente subjetividades sociales o colectivas -estructuras de percepción de la realidad de los grupos humanos-. Es decir, el estructuralismo cree que a cada relación material con la realidad corresponde una cierta percepción de ella, por lo que se interesa en el estudio de esta percepción o construcción social de la realidad que es distinto en grupos humanos con distinto nivel de complejidad socio-económica. Ahora bien, no le interesan las variaciones particulares, individuales dentro del grupo - como sucede con las posiciones más post-modernas y hermenéuticas-, sino los rasgos generales que comparten todos los miembros de cada grupo social, y que les permite sobrevivir eficazmente dadas unas condiciones materiales concretas. Es decir, el Estructuralismo intenta lo que podría calificarse como una interpretación objeti$v a$, pues para él, el sujeto que se analiza no es importante dado que está determinado socialmente, $y$ el que observa tampoco, porque sólo intenta descubir códigos de sentido que le lleven a entender la percepción de la realidad del grupo observado (1).

Puede entenderse así, que dentro de la llamada Arqueología Cognitiva, hay corrientes sustentadas en principios filosóficos muy diversos; pues aunque todas ellas son reflejo de esta conciencia post-moderna en la que la subjetividad va ganando puestos, sin embargo, algunas de ellas -como la Arqueología Procesual-Cognitiva de C. Renfrewsiguen dando prioridad a la Razón Universal y al positivismo, mientras que otras -como la Arqueología Interpretativa de I. Hodder- ponen el peso definitivamente en el relativismo inherente a la determinación subjetiva de cada individuo de su percepción de la realidad.

Personalmente creo que ambas tendencias bloquean la posibilidad de captar fenómenos relacionados con la cognición en sociedades pasadas. Por un lado, la Escuela Procesual y suArqueología Procesual-Cognitiva, con C. Renfrew (1993, 1994) a la cabeza, no pueden escapar a su afán positivista y la consecuente confianza en laverdad del conocimiento. La prioridad que se da a la lógica propia (o modelo científico) que explica la dinámica de cualquier fenómeno que se estudie, impide asignar un papel significativo a la subjetividad de quien ha inventado el modelo de explicación. Digamos que la mente humana se habría limitado, a juicio de los procesuales, a "desvelar" el mecanismo causal que da cuenta de los efectos visibles. Que determinados grupos humanos no hayan sabido o podido desentrañar aún los "secretos" de ciertos fenómenos naturales se debe, exclusivamente, a problemas de capacidad técnica o de interés, por lo que un/a positivista asume que los símbolos de cualquier grupo humano representan siempre el mismo orden de racionalidad, porque tienden a descubrir significados que pertenecen a la realidad; esto sólo significa que se atribuye a todos los grupos humanos la mente y el sentido de la realidad del propio investigador. L. Binford (1965: 204; Renfrew, 1994: 4) despreció los estudios cognitivos por considerarlos "paleo-

(1) F. Criado: “Existe la Arqueología Estructural?”. Conferencia impartida en la Universidad Complutense de Madrid. 3 de Mayo de 1994. Inédita. 
psicología" y aunque C. Renfrew (1993: 248-9) se propone ahora recuperar ese aspecto, lo quiere hacer a través del "método explícito" que siempre reclamó la Arqueología Procesual, rechazando el relativismo de la corriente post-procesual.

Ello le lleva a un modo particular de encarar la cuestión (Renfrew, 1993: 249): los procesuales no estudian qué pensaba la gente-pues creen que eso es paleopsicología-, sino cómo pensaba. Estudian procesos mentales. Por ejemplo, ¿cuándo apareció el lenguaje y cómo? Analizan objetivamente el fenómeno de la cognición desde el punto de vista de cómo se conoce, y distinguen dos campos de actuación: los grupos pre-sapiens y los sapiens. Es cierto que en el primero, aún quedan aspectos por conocer desde un punto de vista positivista, como por ejemplo, ¿cómo avanzan las capacidades intelectuales de los primeros Homo, o la comparación de las del neandertal y el sapiens? Sin embargo, es mucho más difícil analizar cuestiones de este tipo cuando empiezan las culturas del Holoceno. Un ejemplo podría ser la obra de S. Mithen (1996), The Prehistory of the Mind, donde se intentan comprender las transformaciones culturales del pasado vinculándolas con modificaciones del modo en que la mente pudo estar organizada. Por su parte, Renfrew (1993) incluye en esta corriente el estudio de la forma en que se han utilizado los símbolos en diseños, medidas, relaciones sociales (para regular comportamientos) y señala que el avance en este campo es más fácil en Arqueología histórica, lo que resulta fácilmente comprensible.

Por su parte, la Escuela Post-Procesual (o Arqueología Interpretativa, con I. Hodder a la cabeza), al asumir la imposibilidad de escapar a la subjetividad (la mente) del investigador, abandona cualquier intento de aproximación a la percepción que "otros" puedan tener de la realidad. No se trata, en su caso, de negar que esa percepción pueda haber sido diferente, sino de aceptar que es inextricable. Dado que tenemos que utilizar nuestra propia mente para acceder a ese conocimiento, y dado que ésta está culturalmente determinada por nuestra lógica y orden de comprensión de la realidad, nunca podremos acceder de forma libre y abierta al modo en que "otros" han percibido la realidad y han dado sentido a sus símbolos. La única posibilidad de trabajo es el desarrollo de una "narrativa" del pasado, donde sólo cambian el "escenario" de la representación, mientras que la "mente" que se atribuye a sus actores sigue siendo la del investigador -pues ellos mismos reconocen que no podemos escapar de ella-. Es decir, intentan la aprehensión del sentido a través de un pensamiento especulativo, pretendiendo acceder a laintención que el autor de la obra -arqueológica- tuvo al realizarla, mediante la intuición del investigador. Esto es, aplican principios hermenéuticos. De hecho, I. Hodder (1991: 10; 1993: 257) defendió ya explícitamente el fundamento hermenéutico de la Arqueología Interpretativa, en lo que me gustaría detenerme un momento para aclarar por qué la corriente teórica que, en apariencia, más ha hecho por poner de relieve la importancia del estudio de los aspectoș cognitivos del pasado, resulta - a mi juicio-incapaz de profundizar en ellos.

\section{LA HERMENÉUTICA Y LA INTERPRETACIÓN DE LAS SOCIEDADES ORALES}

Sin duda ninguna, la hermenéutica abrió las posibilidades de entender nuestro propio proceso de conocimiento y la cualidad emocional de todo lo intelectualmente significativo para uno mismo.W. Dilthey, profesor de filosofía en Berlín durante la Alemania guillermina de la segunda mitad del siglo XIX, profundizó en el problema de la historicidad y de la comprensión de los hechos históricos, consiguiendo aunar en una síntesis lúcida y explicativa la filosofía, la psicología y la historia (Gadamer, 1994: 36). Dilthey basó su filosofía en la experiencia interna de la comprensión, que muchas veces constituye una totalidad de sentido que va mucho más allá que el mero razonamiento causal a que lleva la concatenación de conceptos. El sentido de la estructura no se formaría en torno a la última vivencia, sino en torno a una vivencia decisiva, crucial por su significación, ya nos refiramos a la trayectoria vital individual o a la secuencia histórica (Gadamer, 1994: 36-37). Es decir, la comprensión en las llamadas Ciencias del Espíritu diferiría estructuralmente, a su juicio, del método cognitivo de las Ciencias Naturales, porque en las primeras el sentido se produce cuando el sujeto es capaz de interpretar, en función de su propia experiencia, el significado global de aquello que estudia.

Reconozco parte de mis estrategias de conocimiento en lo que Heidegger denominó "el círculo hermenéutico" y todo mi discurso inicial podría reconocerse en la declaración de Gadamer (1994: 61) -quien matiza y modifica en parte los planteamientos de Dilthey-, respecto a que "el que reali-

T. P., 56, n. ${ }^{\circ}$ 2, 1999 
za estudios históricos depende de la experiencia que él mismo posea de la historia. Por eso la historia debe escribirse siempre de nuevo, ya que el presente nos define".

Sin embargo, y a pesar de todo ello, creo que la hermenéutica fenomenológica resulta incapaz de comprender los procesos vividos en la Prehistoria porque les atribuye el sentido que para nosotros tiene la realidad, derivado de nuestra propia experiencia. De hecho, el núcleo de la hermenéutica antigua es el problema de la interpretación alegórica $(\mathrm{Ga}-$ damer, 1994: 97). Hacía referencia al arte de traducir, explicar e interpretar, cuando el sentido no era evidente, los textos que conectaban el mundo de los dioses con el de los humanos. Actualmente tiene implícita una especie de conciencia metodológica (Gadamer, 1994: 96) cuyo fin es aclarar el significado de discursos que utilizan un lenguaje distinto del propio. El primer documento en el que la palabra "hermenéutica" se utiliza en el título de un libro data de 1654, fecha desde la que se distingue una hermenéutica teológico-filológica y una hermenéutica jurídica (Gadamer, 1994: 96). En sentido teológico la "hermenéutica" significa "el arte de la correcta exposición de la Sagrada Escritura", y en ella, tanto como en la hermenéutica humanista de la Edad Moderna, el objetivo es "la correcta interpretación de aquellos textos que contienen lo decisivo, lo que es preciso recuperar" (Gadamer, 1994: 97). La hermenéutica intenta dejar a un lado la comprensión tradicional de los hechos pasados para rescatar el sentido original encubierto o desfigurado, para lo que debe acudir a las fuentes originales (Gadamer, 1994: 98).Y el proceso de recuperación del sentido es, siguiendo a Gadamer (1994: 65), el siguiente: "El que intenta comprender un texto hace siempre un proyecto. Anticipa un sentido del conjunto una vez que aparece un primer sentido en el texto. Este primer sentido se manifiesta a su vez porque leemos ya el texto con ciertas expectativas sobre un determinado sentido. La comprensión del texto consiste en la elaboración de tal proyecto, siempre sujeto a revisión como resultado de una profundización del sentido". Es decir, "la anticipación del sentido, que involucra el todo, se hace comprensión explícita cuando las partes que se definen desde el todo definen a su vez ese todo" (Gadamer, 1994: 63), marcando con ello la relación circular propia de la hermenéutica.

Si me he querido detener en este punto es porque, como digo, la hermenéutica constituye la base más generalizada sobre la que sustentar filosófica- mente los últimos intentos de acceder a aspectos cognitivos del pasado pero, como espero haber dejado suficientemente claro, la hermenéutica es una praxis de traducción e interpretación de textos, cuyo sentido último intenta recuperar. Es decir, la hermenéutica surge como un método de interpretación histórica en el que el sentido que desde el presente tiene la historia puede ayudarnos a entender los procesos ocurridos a lo largo de su desarrollo. Pero se trata siempre de sociedades con textos, y es aquí donde yo creo que reside una de las confusiones más importantes respecto a las posibilidades de su aplicación a las sociedades prehistóricas, ya que, por definición, éstas no generaron textos. El argumento de ninguna manera convencerá a los defensores de la Arqueología Interpretativa que, como de sobra sabemos (Shanks y Tilley, 1987, por ej.), decidieron considerar que el registro arqueológico constituía un texto a la espera de ser descifrado, por lo que cabía "leer" sus significados como en cualquier texto compuesto por palabras. Los objetos son símbolos, y su mera elaboración ya constituye un cierto tipo de lenguaje que está sujeto a "interpretación" como cualquier texto literario.

Sin embargo, a mi juicio, semejantes conclusiones no pueden sostenerse desde el rigor filosófico, puesto que la escritura constituye un modo de representación de la realidad específico que implica una cierta percepción de la realidad por parte de la sociedad que la produce y por tanto, un modo de relacionarse con ella que es estructuralmente distinto al que poseen las sociedades orales. Es decir, creo que interpretar desde la hermenéutica fenomenológica el pasado prehistórico no lleva sino a proyectar la base común de experiencia de la realidad que comparten las sociedades con escritura a las que poseen un tipo muy distinto de experiencia, y por tanto, de sentido de la realidad. De otra forma: la interpretación hermenéutica exige una base común de experiencia, un horizonte de intelegibilidad que yo creo que no comparten las sociedades ágrafas y las literarias.

Lo que quizá no se comprende desde las posiciones hermenéuticas es que la experiencia de la realidad está culturalmente determinada, no es abierta y aséptica; nosotros no podemos elegir percibir cualquier realidad; no la seleccionamos en términos absolutos, sino que la cultura predetermina qué porción va a constituir "la realidad" para cada uno de sus miembros, ya que la realidad -entendida como el conjunto de la naturaleza humana y no-humana y las dinámicas en las que se insertan- es incommensurable e inabarcable para la 
mente humana. Éste es el punto esencial que diferencia a las posiciones hermenéuticas de las estructuralistas, que intentan por ello desentrañar las estructuras básicas de ordenación del mundo y comprensión de la realidad de cada grupo humano, lo que para cada uno de ellos, colectivamente, constituye "la realidad".

Cada sociedad selecciona la porción de realidad que puede contemplar en función del control material que haya desarrollado sobre ella, por lo que no podemos pensar que la realidad es una y la misma para todos los grupos humanos. El modo en que se realiza semejante selección consiste en ordenar sólo parte de la desordenada realidad, poniendo en relación los hechos y fenómenos que se van a contemplar con dos parámetros esenciales: el Tiempo y el Espacio.

\section{TIEMPO Y ESPACIO COMO CUALIDADES DEL ORDEN QUE ATRIBUÍMOS A LA REALIDAD PARA PODER COMPRENDERLA}

La realidad es demasiado compleja como para que podamos hacernos cargo de su totalidad; la angustia que se derivaría de nuestra impotencia, de la conciencia lúcida de la pequeñez esencial de lo humano, impediría el desarrollo de recursos que garantizaran nuestra supervivencia.Y sin embargo, ningún grupo humano ha muerto de angustia, que se sepa, ni ha sido incapaz de generar estrategias tan exitosas como variadas para sobrevivir en cualquier medio que podamos imaginar. De hecho, como ya apuntara Elías (1990b) la confianza en que somos capaces de hacernos cargo de las circunstancias en las que nos ha tocado vivir es fundamental para desarrollar sistemas de control de esas circunstancias. Ahora bien, el control real de las condiciones materiales de vida puede ser muy variado, y en ocasiones -como en las sociedades de cazadores-recolectores-, ciertamente reducido. Sin embargo, no hay sociedades que tengan una mayor sensación de control que otras, que se consideren más seguras que otras, que sufran de miedo e impotencia más que otras. Esto es así porque los grupos humanos seleccionan la experiencia que son capaces de asimilar y lo hacen a través de la configuración particular de los dos parámetros básicos de orientación en la realidad: el Tiempo y el Espacio.

Tiempo y Espacio no son realidades dadas. No existe algo como el Tiempo, o como el Espacio, que se pueda tocar, poseer o delimitar (Gell, 1996: 231). Tiempo y Espacio son cualidades de nuestra percepción de la realidad, referencias de orden que nos permiten clasificar y de esa forma asimilar los de otro modo desordenados hechos de la experiencia. La experiencia, para ser, tiene que estar ordenada porque si no, no la podríamos asumir, ni relatar, ni utilizar para seguir construyendo nuestra vida. Si no pudiéramos conferirle un cierto orden y un mínimo sentido, sólo tendríamos inputs sensoriales que no tendrían cabida en un esquema de comprensión o asimilación, lo que significaría nuestro enloquecimiento, además, desde luego, de nuestra incapacidad para desarrollar modos operativos de actuación material sobre la realidad. Es decir, nuestra percepción del Tiempo y el Espacio no es resultado de nuestra experiencia, no tenemos una idea de lo que es el Tiempo y el Espacio porque hayamos tenido ciertas experiencias, sino que tenemos ciertas experiençias porque tenemos una cierta percepción espacial y temporal de cualquier hecho de la realidad que se nos transmite al nacer para que podamos comprender esa realidad. Ésta es la diferencia esencial entre las posiciones hermenéuticas y las estructuralistas: las primeras creen que el individuo construye su realidad, mientras que las segundas creen -y yo con ellas- que la realidad, y con ella el individuo, están socialmente construídas. Por eso creo que existe una relación, estructural y directa, entre percepción de la realidad -de la que se ha seleccionado ordenándola espacial y temporalmente- y complejidad socio-económica; por eso creo que se puede investigar, y por eso considero tan estéril e improcedente generalizar nuestra propia percepción a las sociedades de la Prehistoria.

El problema que tenemos en Historia y a mi juicio, desde luego en Prehistoria, es que confundimos la referencia de orden con la realidad que ordenamos, y atribuímos a la realidad lo que pertenece a nuestra percepción de ella. Esto no tendría mayor importancia si no fuera porque lo que hacemos al ordenarla es seleccionar la realidad que podemos contemplar. La consecuencia de esto es que si no somos conscientes de los problemas de delimitación ontológica en que nos movemos, atribuiremos $-\mathrm{y}$ de hecho atribuimos-a los grupos de la Prehistoria una percepción de la realidad como la que a nosotros nos caracteriza, incurriendo en un error que imposibilita, de entrada, comprender la realidad en la que ellos se movieron. Para demostrar que nuestro modo de percepción de la realidad, y por tanto, delTiempo y el Espacio, es particular de nuestra cultura, comen- 
zaré por analizar la determinación cultural de nuestro propio sistema de identidad.

\section{CONCEPTOS DE "INDIVIDUO" $Y$ "SOCIEDAD”. EMPEZANDO POR EL PRINCIPIO}

El concepto de "individuo" hace alusión a una entidad aislada, un elemento que tiene identidad propia, cuya existencia se puede concebir en sus propios términos. Pues bien, el término "individuo" no se empezó a aplicar a las personas hasta el siglo XVII (Elías, 1990a: 185), en prueba de que hasta entonces éstas no se consideraron a sí mismas susceptibles de dicha identidad. En latín clásico no existía el término individuum y aunque existía el de "persona", presentaba un grado bajo de generalización (Elías, 1990a: 184), refiriéndose sólo a una categoría de derecho -junto a las res y las actiones(Mauss, 1991: 323). En el latín medieval, las palabras individualis o individuus se utilizaban para hacer referencia a algo indivisible, inseparable. Se aludía a la especificidad de cada caso particular de una especie, advertida por los escolásticos (Elías, 1990a: 185-186). Y aunque desde el punto de vista de las instituciones y el derecho parece poder situarse el germen del desarrollo constitucional y de la emergencia del individuo en el paso del siglo XII al XIII, momento de la aparición del "ciudadano" -tanto de esta vida como de la otra, como demuestra a su vez el nacimiento del Purgatorio (Le Goff, 1981: 268-269)-, sólo a partir del Renacimiento empieza a aplicarse el concepto "individuo" a las personas (Elías, 1990a: 185). ¿Por qué? Parece obvio que porque sólo en este momento el desarrollo de las sociedades occidentales había desembocado en un tipo de estructura y situación en la cual la percepción del ser humano sobre sí mismo le hacía concebirse como algo que podía ser independiente y distinto de los demás, como un agente de acciones particulares que podían diferenciarse de las colectivas, como alguien suficientemente seguro del control sobre las circunstancias en las que vivía como para poder concebirse como una parte aislada del grupo protector (sea la familia, el clan, la tribu o el Estado) sin el que, hasta ese momento, se podía concebir. Y le hizo falta una palabra para designar la nueva percepción de sí mismo (2).

(2) Indudablemente, esta percepción de uno mismo como una entidad aislada de realidad se había desarrollado ya entre algunos sectores de la población en las grandes civilizaciones clá-
Por ello, esta percepción individualizada de nosotros mismos no puede generalizarse a otras culturas. Al parecer, en todas ellas existe una palabra para denominar a uno mismo, al "yo", así como sufijos y mecanismos verbales que expresan la relación entre el sujeto que habla y el objeto del que habla (Mauss, 1991: 310; Elías, 1990a: 123). La cuestión es que la representación, el concepto, la comprensión de ese "yo" es distinta, dependiendo entre otros factores, del nivel de complejidad socioeconómica. En aquellos grupos donde la economía y la política no constituye una esfera escindida de la malla social (Dumont, 1987: 18; Clastres, 1987: 111-112) y a los que Lévi-Strauss (1964) atribuyó un tipo de pensamiento "salvaje", el grupo supone hasta tal punto la garantía de supervivencia de cada uno de sus miembros, que éstos no se pueden concebir sin aquel. La identificación que se hace con el grupo que le da protección y seguridad es tal, que la percepción de uno mismo es mucho más colectiva y social que individual (Elías, 1990a: 196; Dumont, 1987; Mauss, 1991: 321).

Por su parte, la "sociedad" es sólo el conjunto de interrelaciones de los seres humanos que la componen. Por tanto, hacer referencia a sociedades distintas es hacerlo al establecimiento de relaciones diferentes entre sus miembros. Pero las relaciones no son algo superpuesto a las personas, ajeno a su propia constitución como seres humanos (Elías, 1990a), sino la expresión, a distintos niveles, de sus funciones psíquicas. Para poder sobrevivir, todos los humanos hemos desarrollado dos tipos de funciones: físicas y psíquicas. Las primeras son inmutables, pues el corazón, los pulmones o el estómago tienen en todos los mismos procesos y ritmos; pero las segundas varían dependiendo del contexto de conocimiento que caracterice a cada sociedad particular, pues para darse necesitan del aprendizaje

sicas. Es decir, allí donde la división de funciones y especialización del trabajo habían revestido suficiente complejidad como para permitir el surgimiento de personas con conciencia de su diferencia respecto al resto de los componentes del grupo social, en un grado mayor que de las semejanzas que a ellos les unía. Como intentaré explicar a continuación, esto implica, en sí mismo, una relación menos conectada emocionalmente con la realidad a la que, por lo tanto, se intenta comprender en términos lógicos. Los grandes filósofos clásicos son, este sentido, claros ejemplos de los que yo llamaría "primeros individuos de la Historia", lo que permite entender que la reflexión en torno al tema pueda remontarse hasta Aristóteles, retomándose después en la filosofía del siglo XII y XIII, momento histórico en el que a través de la burguesía, como digo, se comienza a generalizar la existencia de individuos. Sin embargo, esta percepción no se generalizará en la sociedad hasta el comienzo de la Modernidad, en que la complejidad socio-económica sitúa a cada miembro del grupo social en una posición diferente desde la que contemplar la realidad. 
y la relación. Es decir, cuando hacemos referencia a la existencia de sociedades diferentes, lo que estamos diciendo en realidad es que sus miembros tienen una modelación distinta de las funciones psíquicas (manteniendo siempre la misma capacidad lógica y emocional) que se traduce en relaciones diferentes entre sí. O lo que es lo mismo: que tienen distintos órdenes de racionalidad -si por este concepto entendemos el conjunto de las pautas afectivas e intelectuales de un grupo humano-, o modos distintos de percepción de la realidad.

Como ya empecé a señalar párrafos atrás, los distintos grupos humanos creemos que controlamos en medida suficiente el mundo en que vivimos, cuando en realidad lo hacemos en medidas muy distintas, y nunca, nunca, suficientes. Sin embargo, todos nos sentimos seguros en el mundo en el que nos ha tocado vivir, lo cual constituye la prueba evidente de que construímos socialmente la realidad que percibimos. Es decir, la percepción que las funciones psíquicas determinan y por las que se ven determinadas, debe ser coherente con la relación material con la realidad que cada grupo humano establece para permitir una supervivencia efectiva, de lo que cabe deducir una relación estructural entre percepción de la realidad y control material de la realidad, lo que significa decir entre modelación de la conciencia (funciones psíquicas = pautas afectivas e intelectuales) y complejidad socio-económi$c a$ en cada grupo humano.

Atendiendo a esta relación, podríamos resumir el modo en que los grupos de escasa complejidad socio-económica (entendiendo por tal una reducida división de funciones y especialización del trabajo) construyen su identidad del modo esquematizado en la figura 1.

En la tradición occidental, comenzando en el siglo XII como decía antes -momento de aparición de la burguesía como tercer estamento social y de su progresión social a través de mecanismos individualizadores-, empieza a abrirse paso un modo distinto de percepción de la realidad (Olson, 1994: 61), lo que incluye, obviamente, tanto a uno mismo como a todo el conjunto de elementos y dinámicas que nos rodean. Este nuevo modo de percepción llega a su plena conformación cuando el contexto socio-económico exige -y es resultado de, en una relación de mutua determinación-la existencia de individuos conscientes de sus deseos y de su capacidad de satisfacerlos como agentes sociales. Es decir, cuando el capitalismo sea el resultado -y exija la existencia- de personas consumidoras y traba-

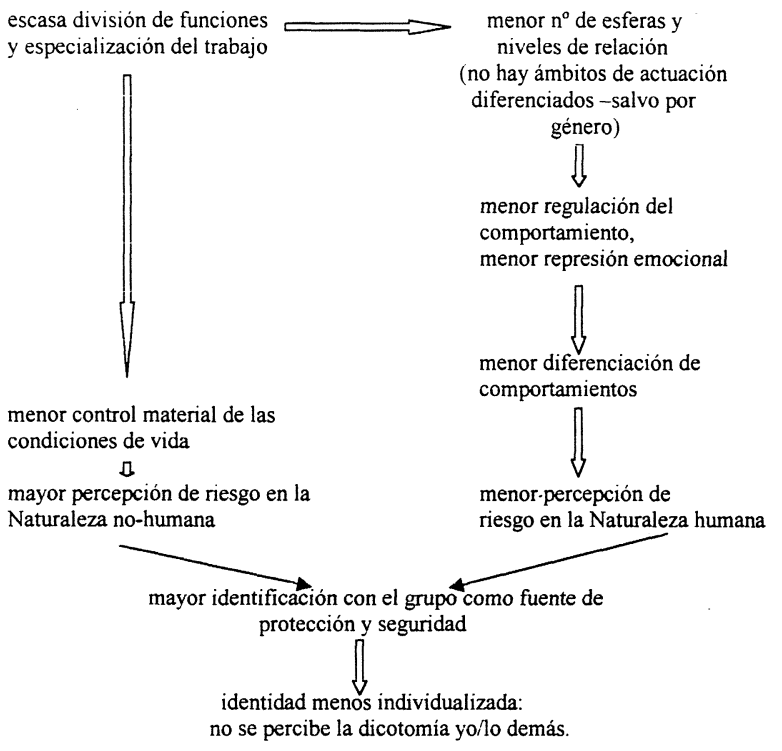

Fig. 1. Mecanismo básico de construcción de la identidad en grupos de escasa complejidad socio-económica.

jadores a cambio de un salario con que satisfacer sus necesidades de consumo. Este estado de cultura se ha dado en llamar Modernidad. Esto es, a partir de la Modernidad, la percepción de la realidad se caracteriza por dos rasgos complementarios: el desarrollo de la individualidad y la prioridad, como forma de relación con la realidad, del conocimiento científico -aunque siempre se mantendrá también la forma más emocional, menos individualizada, según de qué sectores del grupo social se trate o de qué esferas de la realidad hablemos-.

El modo de identidad que corresponde a las personas que se relacionan de este modo con la realidad será, por tanto, muy diferente, y se basará, esencialmente, en el mecanismo reflejado en la figura 2.

Es decir, aquí la ficción de que controlamos las circunstancias en las que vivimos hasta un punto suficiente no viene ya dada por la seguriđad que confiere el grupo, sino por el alejamiento emocional respecto a esas circunstancias -la confianza en la existencia de un "yo" separado de lo demás-y por el grado de predicción del modelo de representación que utilicemos. Ello conduce a una necesidad ilimitada de perfeccionar constantemente esos modelos, de profundizar cada vez más detallada y analíticamente en los vínculos causales que los pueden explicar, lo que no es sino decir que al tiempo que la subjetividad gana campos en nuestra percepción de la realidad, lo hace también el desarrollo de los modelos científicos y la inversión económica destinada a promoverlos. 


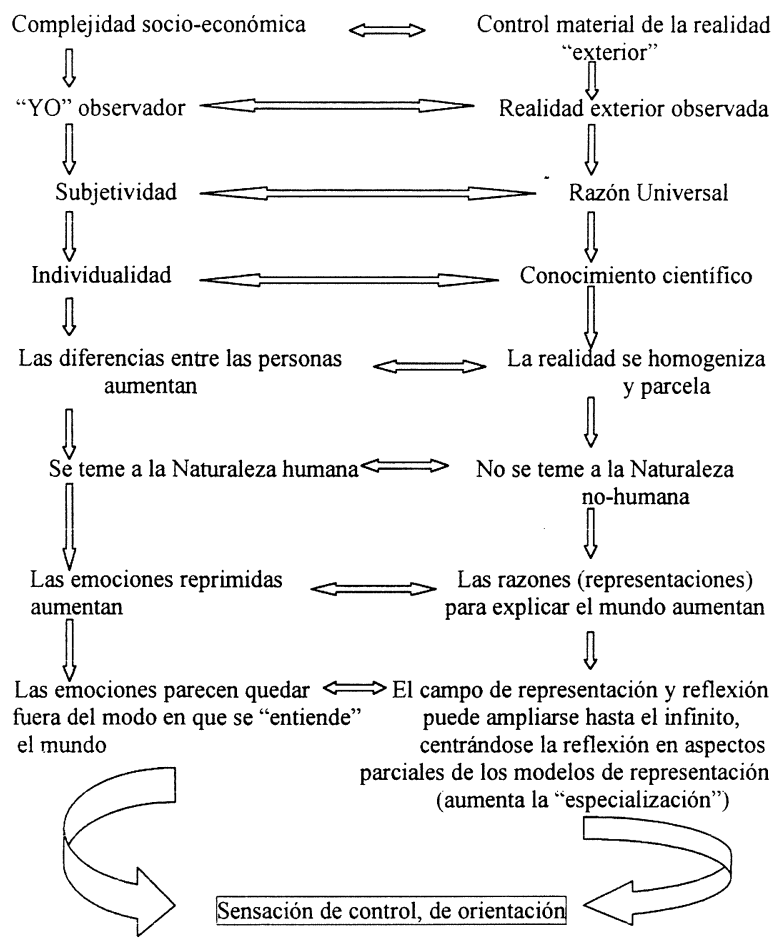

1) Seguridad por alejamiento emocional ("Yo" separado de lo demás) 2) Seguridad por la exactitud del modelo de representación: necesidad de perfeccionamiento constante del modelo (Razón Universal)

Fig. 2. Mecanismo básico de construcción de la identidad a partir de la Modernidad.

\section{Relación entre construcción social de la realidad y percepción del Tiempo y el Espacio}

Como habíamos empezado a ver, la "realidad"se selecciona a través de dos ejes básicos de ordenación: el Tiempo y el Espacio; estos dos parámetros determinan la porción de realidad que cada grupo humano va a contemplar como existente, la que va a tener en cuenta; ambos establecen relaciones posicionales entre hechos observables, y la única diferencia entre ellos es que el Espacio pone en relación los hechos observables con referencias inmóviles, y el Tiempo con referencias móviles, aunque de movimiento recurrente (los ciclos del sol, de la luna o los movimientos de las manecillas de un reloj) para que puedan servir como principio de ordenación (Elías, 1992: 98-99). Sólo aquellos hechos que se ponen en relación con alguna de estas dos referencias son contemplados como parte de la realidad existente para un grupo humano, ya que es la única ordenada, por lo que resulta tan importante comprender los mecanismos que utilizamos para construirlas.Y puesto que defiendo una relación es- tructural entre modo de percepción de la realidad y complejidad socio-económica de un grupo humano, asumo consecuentemente una relación estructural entre las pautas básicas de percepción delTiempo y el Espacio y dicho grado de complejidad. $\mathrm{La}$ primera hipótesis que se deriva de ello es la afirmación de que los grupos de la Prehistoria no podían percibir el Tiempo o el Espacio como nosotros lo hacemos, por lo que la realidad que podían conocer era distinta de la que nosotros contemplamos.

Para comprender los mecanismos a través de los cuales construimos socialmente la realidad en la que vivimos, he utilizado (cfr. también Hernando, 1997; e.p.) la distinción que hace Olson (1994) entre los dos modos esenciales en que los humanos pueden representar la realidad: la metonimia y la metáfora. La metonimia utiliza signos que están contenidos en la realidad que representa, mientras que la metáfora utiliza signos arbitrarios, ajenos, externos a la realidad que representa. En este sentido, por ejemplo, el nombre de dios o una estatua sacra o una bandera son representaciones metonímicas -de lo que se derivan los pecados de blasfemia, el de utilizar el nombre de dios "en vano", el sacrilegio de la mutilación de los "santos" o el delito de ultraje a la patria, pues atacando la representación se ataca la realidad a la que representa $(\mathrm{Ol}-$ son, 1994: 167)-, mientras que la escritura o una fórmula matemática son representaciones metafóricas, por cuanto utilizan signos arbitrarios para representar la realidad. Aquí se puede cambiar el signo sin cambiar la realidad, mientras que en el caso de los signos metonímicos cambiará la realidad si cambiamos el signo. En este sentido, el Mito es una representación metonímica de la realidad, mientras que la Ciencia es una representación metafórica.

Todas las sociedades utilizan ambos modos de representación, pero dan prioridad a uno de ellos. A mi juicio, se establece una relación directa entre control material de la realidad y modo de representación: se representa metonímicamente aquella parte de la realidad a la que no se controla y cuya dinámica de funcionamiento no puede explicarse, por tanto, conforme a una lógica abstracta -representada a través de símbolos o ecuaciones, de hipótesis o leyes-que le dé sentido.Y es precisamente porque no puede explicarse así-representarse asípor lo que se la teme. Por ello, cuanto menor es el control material sobre la realidad, es decir, la complejidad socio-económica de un grupo humano, mayor es el uso de representaciones metonímicas 
para representar su realidad. En consecuencia, los grupos cazadores-recolectores o agricultores de roza suelen utilizar como signos de representación elementos que, a su vez, forman parte de la realidad en la que viven: los montes, las nubes, los árboles, los ríos. Su modo de relación con la realidad no ha desarrollado la distancia que permitiría elaborar modelos científicos de explicación -interponer signos metafóricos de representación- para comprender la lógica que guía sus dinámicas, y por lo tanto, la temen. La única lógica (¿?) que conocen es la del comportamiento humano, por lo que la atribuyen al funcionamiento de cualquier aspecto de la Naturaleza no-humana a la que, además, sacralizan, dado su poder de dar y quitar la vida. Por eso la vida se explica en función de los caprichos o generosidades de los montes o las nubes que, además, hablan entre sí y se comunican permanentemente con los humanos cuyas vidas determinan. Esto es el Mito (Hernando, 1997).

Por su parte, existe una relación directa entre desarrollo del individualismo y el modo de representación, pues a medida que se percibe una distancia entre un "yo" y el resto de la realidad, ésta se podrá explicar a través de dinámicas que le son propias -la Ciencia- y que implican la utilización de símbolos arbitrarios, esto es, metafóricos -las matemáticas, física, hipótesis sociológicas, económicas, antropológicas, etc.-. Es decir, creo que no solamente en términos sociales, sino también en términos personales, un aumento del individualismo se corresponde con un aumento de la representación metafórica de la realidad. Pero a pesar de que estas diferencias puedan detectarse entre los distintos colectivos de una sociedad definida por tanta diversidad de funciones.como la nuestra-no es igual la percepción de la realidad de un campesino que de un profesor de astrofísica-, cada sociedad como conjunto enfatiza uno de ambos modos para la construcción colectiva de la realidad en la que vive. Por eso, las sociedades menos divididas o complejas dan prioridad al modo metonímico, mientras que las más complejas al metafórico. Sin embargo, ambos existen en ambas, y al igual que en nuestra sociedad la metonimia se utiliza, por ejemplo, para representar la muerte, esfera aún no explicada y sobre la que, desde luego, no se posee sensación de control -y ya sabemos la forma humana de varón adulto del dios católico y las iras y generosidades que le pueden caracterizar-, en sociedades orales, sin división de funciones o especialización del trabajo, la metáfora se utiliza también en determinadas ocasiones. Sin embargo, como Olson (1994: 141) señala, mientras en nuestra sociedad utilizamos la metáfora en cualquier género de discurso -nos movemos a través de mapas, comerciamos con el concurso de cálculos matemáticos y nos comunicamos a través de la escritura-, en las sociedades orales la metáfora sólo se utiliza en determinados géneros, como la oratoria o la poesía, propios no de la vida cotidiana sino de ocasiones ceremoniales. Y además, los discursos que así se construyen están siempre sujetos a interpretación. Es decir, hay sociedades donde la representación metonímica es la generalizada para la construcción y dinámica colectiva de la realidad, y hay otras donde el modo generalizado es la metáfora, aunque en todas se utilicen ambas en alguna esfera de actuación determinada. Esto es así porque todos los grupos humanos tenemos la misma lógica, y somos capaces de los mismos modos de representación. Por ello insisto en que no se trata de capacidades o de diferencias intrínsecas, sino de modos de supervivencia psíquica en función de los sistemas socio-económicos, de maneras distintas de contemplar la realidad dependiendo de lo que podamos hacer con ella.

¿Qué tiene esto que ver con el Tiempo y el Espacio? En una representación metonímica de la Naturaleza humana y no-humana, la realidad que se representa contiene los símbolos para hacerlo. Luego para representar el Tiempo se elegirán fenómenos dinámicos de movimiento recurrente contenidos en la realidad: mareas marinas, movimientos del sol o de la luna, etc., y para representar el Espacio -o lo que es lo mismo, para ordenar espacialmente la realidad-, elementos inmóviles de la $\mathrm{Na}$ turaleza: árboles, montañas, ríos o rocas. Es decir, en una representación metonímica de la realidad, propia de las sociedades escasamente complejas en términos de división de funciones o de especialización del trabajo, el Espacio se confunde con la "Naturaleza no-humana inmóvil" y el Tiempo con la "Naturaleza no-humana de movimiento recurrente", vivenciada a través de la actividad. Esto es así porque:

a) el único "espacio" que puede contemplarse es el que forma parte de la experiencia (al estar contenido el símbolo en la realidad, sólo puede ordenarse la realidad que se conoce, la que se experimenta vivencialmente). El resto es caos (no está ordenado, no puede ordenarse, porque para ordenarlo hay que tomar parte de sus elementos como símbolos, es decir, hay que conocerlo), luego no se contempla. Lo mismo sucede con el Tiempo, de lo 
que se deriva que sean grupos humanos que tienden a vivir sobre todo en el presente, pues sus modos de representación no dan cabida -y no incentivan el interés- por aquello que no es acogido por los ritmos marcados por los fenómenos naturales que sirven de referencia. Por eso, su presente puede llegar a ser un presente amplio, que incluya toda la variación recurrente inherente, por ejemplo, a cada ciclo estacional, pero varían profundamente el sentido del pasado y del futuro lejano, que pueden no incluirse en modo alguno en el sistema de ordenamiento de la realidad.

b) El espacio está referido a elementos heterogéneos (ese árbol, aquella montaña,...), al confundirse el símbolo y la realidad. Por ello, el hecho de que un elemento de la Naturaleza no-humana pueda considerarse símbolo de ordenación espacial o temporal exige que forme parte de la experiencia personal. De ahí que el Espacio en estos grupos no sea transformable, pues al quitar un árbol se anula un símbolo también, lo que llevaría a la desorientación. El Espacio es, en los grupos de escasa complejidad socio-económica, su contenido (al contrario que en nuestra cultura, donde llamamos "espacio" al continente, a los límites).

Por el contrario, en una representación metafórica, como la que caracteriza a la sociedad moderna-occidental, Tiempo y Espacio proveen de una representación y ordenación de los hechos de la experiencia a través de modelos interpuestos, de referencias superpuestas. Es decir, refieren los hechos que ordenan a modelos pensables en sí mismos como entidades distintas de la realidad que ordenan. Los símbolos con que se representa la realidad no están contenidos en ella: para el Tiempo se utilizan relojes o calendarios; para el Espacio, mapas, delimitaciones político-administrativas, etc.. Es decir, en el modo de percepción que corresponde a sociedades complejas e individualizadas:

1. Los contenidos del Espacio o del Tiempo se homogenizan a través de su representación simbólica con signos de igual peso representativo. En un mapa o en un calendario cada uno de los símbolos tienen el mismo valor, pues la relación que establecemos con ellos no es emocional.

2. El significado no pertenece a los elementos que se ordenan, sino a los símbolos con que se representan, lo que hace que el Espacio y el Tiempo tengan significados transformables. Nos sentimos orientados cuando somos capaces de imaginar una representación de nosotros mismos en un modelo de representación de la realidad-sabemos dónde estamos cuando nos localizamos en un mapa o en un calendario o en un reloj-. Por eso puede destruirse la Naturaleza no-humana, podemos quemar los montes o alterar las montañas sin que ello haga que nos sintamos desorientados en la realidad. Sin embargo, y a cambio, para sentirnos seguros necesitamos ampliar constantemente el modelo de representación, pues es con él con quien establecemos la relación. Y de ahí el desarrollo de sistemas geográficos, satélites, fotografías de subsuelos, mapas del Universo, viajes interplanetarios, etc., para que podamos seguir manteniendo nuestra sensación de seguridad y orientación en el Espacio, y el perfeccionamiento insaciable de los sistemas de cronología o de los detalles y límites de la Historia para que nos suceda lo mismo en relación al Tiempo.

Se me dirá, con razón, que la cultura modernaoccidental no sólo presenta este modo de relación con el Tiempo y con el Espacio, sino que aún mantenemos una vinculación emocional con ciertos lugares, o que determinados hechos son valorados en términos temporales de distinta manera, según la "carga" emocional que representaran para nosotros. Y es cierto. Como antes señalaba, los modos metonímicos de representación no se abandonan necesariamente porque se adopten prioritariamente los metafóricos, por lo que, como Entrikin (1991: 1) afirmaba -refiriéndose al Espacio-, existe una bipolaridad en nuestra percepción del tiempo y el espacio que hace que nos sintamos el centro de un mundo al que sabemos sin centro, mientras que los grupos de escasa complejidad socio-económica ajenos a esa paradoja, se sienten el centro de un mundo que creen centrado en ellos mismos.Y esto tanto en términos de Tiempo como de Espacio: la importancia que damos en términos emocionales a lo que nos sucede en el presente en nuestra cultura es contradictorio con la prioridad que, en términos racionales damos al futuro, donde depositamos toda la expectativa de nuestra vida; sin embargo, los grupos de escasa complejidad, viven esencialmente en el presente en términos emocionales y racionales.

Todo esto lleva a una última consecuencia: aquellas sociedades que son más complejas y tienen un mayor control material de las circunstancias de vida valoran el cambio como parte esencial y positiva de la lógica social. Sin embargo, los grupos cuyo control material de la realidad es reducido, asumen siempre el cambio como una amenaza, pues dada su escaso dominio y entendimiento de las causas que provocan los fenómenos naturales, sólo tienen seguridad de supervivencia en las condiciones que ya 
conocen. De ahí que se resistan a transformarse. Esto hace que éstos últimos privilegien el Espacio, el parámetro más estático, como eje esencial sobre el que ordenar la realidad, mientras que la cultura moderna-occidental da prioridad al Tiempo, más dinámico y por tanto estructuralmente más coherente con la posibilidad de introducir hechos nuevos de experiencia.

\section{CONCLUSIÓN}

La conclusión general parece clara: no podemos entender a los grupos de la Prehistoria si aplicamos los principios positivistas de la Arqueología procesual, o los subjetivistas de la post-procesual puesto que, como vimos al principio, ambos proyectan al pasado la propia mente del investigador y como acabamos de ver ahora, las sociedades del pasado y la nuestra deben haber estado guiadas por lógicas o pautas de racionalidad o modos de percepción y relación con la realidad distintas. Creo que es necesario aplicar principios estructuralistas al estudio de aspectos cognitivos del pasado, pues a menos que asumamos que el comportamiento material de un grupo humano exige un entendimiento del mundo y de la realidad coherente con él para que la supervivencia pueda ser eficaz, será imposible establecer un marco de comprensión de las sociedades de la Prehistoria y de la lógica que guió cualquiera de sus actuaciones.

No se puede comprender la Historia desde el punto de vista del individuo, a mi juicio, porque $e l$ individuo es en sí una construcción social que sólo se inició a partir de determinado momento histórico. Por tanto, pretender que nuestra "intuición" puede constituir una buena base desde la que partir para interpretar los restos del pasado, como hacen los últimos intentos hermenéuticos, implica ignorar que las pautas intelectuales y emocionales -y por tanto la intuición- de cada grupo humano están culturalmente constituidas.

Como digo, el estructuralismo ha venido defendiendo estos principios desde hace ya bastantes años (cfr., por ejemplo, Criado, 1993; Criado y Villoch, 1998). Pero no parece suficiente, dado el peso de la Arqueología anglosajona en el panorama de los estudios prehistóricos y los problemas que la corriente post-procesual presenta en sus fundamentos filosóficos. Sin embargo, creo que es importante seguir reivindicando los argumentos estructuralistas, pues quizá sea la única manera de asumir que el relativis- mo debe estar implicado en el estudio de las culturas, sin que ello conduzca a nihilismo metodológico alguno: no es posible interpretar las culturas del pasado sin tener en cuenta sus formas específicas de percepción de la realidad, pero esas formas, a diferencia de lo pretendido por los post-procesuales, son accesibles, pues son coherentes con el grado de complejidad socio-económica. Hay formas, estructuras generales, que pueden suponerse a distintos grupos humanos con similares grados de complejidad, más allá de las características particulares que pueda alcanzar luego la formulación concreta de mitos o de ritos en cada una de ellas. Hay una ordenación y una selección básica de la realidad que $s e$ puede conocer en términos objetivos, porque resulta contrastable entre la diversidad de grupos humanos que hoy existe. Y creo que esto marca un prometedor y fructífero campo de estudio (3).

\section{BIBLIOGRAFÍA}

Barret, J.C. (1994): Fragments from Antiquity. An Archaeology of Social Life in Britain, 2900-1200 BC. Blackwell. Oxford.

BINFORD, L.R. (1965): "Archaeological systematics and the study of culture process". American Antiquity 31, 2: 203-210.

Bradley, R. (1998): The Significance of Monuments. On the shaping of human experience in Neolithic and Bronze Age Europe. Routledge. London.

Clastres, P. (1987): Investigaciones en Antropología Política. Gedisa. Barcelona.

CRIADO, F. (1993): "Visibilidad e intepretación del registro arqueológico". Trabajos de Prehistoria, 50: 39-56.

Criado, F. y Villoch Vázquez, V. (1998): "La monumentalización del paisaje: percepción y sentido original en el megalitismo de la Sierra de Barbanza (Galicia)". Trabajos de Prehistoria, 55(1): 63-80.

Dumont, L. (1987): Ensayos sobre el individualismo. Alianza Editorial. Madrid.

Elías, N. (1990a): La sociedad de los individuos. Ensayos. Península/Ideas. Barcelona.

- (1990b): Compromiso y distanciamiento. Península/ Ideas. Barcelona.

- (1992): Time: An Essay. Basil Blackwell. London.

- (1993): El proceso de la civilización. Investigaciones sociogenéticas y psicogenéticas. Fondo de Cultura Económica. Madrid.

(3) Este trabajo forma parte de la investigación desarrollada gracias a la concesión del proyecto PB-0276 de la DGES para el "Estudio de la correlación entre construcción de la identidad y complejidad socio-económica en los grupos humanos". Agradezco los comentarios críticos de los evaluadores del artículo.

T. P., 56, n. ${ }^{\circ}$ 2, 1999 
ENTRIKIN, J.N. (1991): The Betweenness of Place. Towards a Geography of Modernity. John Hopkins Universty Press. Baltimore, Maryland.

ERchark, G.M. (1992): The Anthropology of Self and Behavior. Rutgers University Press. New Brunswick, New Jersey.

Gadamer, H.-G. (1992): Verdad y Método II. Ediciones Sígueme. Salamanca.

Gell, A. (1996): The Anthropology of Time. Cultural constructions of temporal maps and images. Berg. Oxford.

GELLNER, E. (1995): “Interpretive anthropology". En I. Hodder, M. Shanks, A. Alexandri, V. Buchli, J. Carman, J. Last and G. Lucas (eds.): Interpreting Archaeology. Finding meaning in the past. Routledge. London: 48-50.

HeRnANDO, A. (1997): "Mitos, metáforas y miedos: sobre la Prehistoria y sus habitantes". Complutum, 8: 247-260.

- (ep.): "El espacio no es solamente un lugar. En torno al concepto de espacio y a sus implicaciones en el estudio de la Prehistoria". Arqueología Espacial, 21.

Hodder, I. (1991): "Interpretive archeology and its role". American Antiquity, 56(1): 7-18.

- (1993): "Social Cognition". Cambridge Archaeological Journal, 3(2): 247-270.

Hodder, I.; Shanks, M.; Alexandri, A.; Buchli, V.; CarMAN, J.; LAST, J. and LUCAS, G. (eds.) (1995): Interpreting Archaeology. Finding meaning in the past. Routledge. London.

LE Goff, J. (1981): El nacimiento del Purgatorio. Taurus Ediciones. Madrid.

LÉvi-STRAuss, C. (1964): El pensamiento salvaje. Fondo de Cultura Económica. México.
MAUSS, M.(1991): "Sobre una categoría del espíritu humano: la noción de persona y la noción del «yo»". Sociología y Antropología. Ed. Tecnos. Madrid: 153-263.

McGuire, R.H.(1992): A MarxistArchaeology. Academic Press. San Diego, California.

Mrthen, S. (1996): The Prehistory of the Mind. A search for the origins of art, religion and science. Thames and Hudson. London.

OLSON, D.R. (1994): The world on paper. The conceptual and cognitive implications of writing and reading. Cambridge University Press. Cambridge.

Renfrew, C. (1993): "Cognitive Archaeology: Some Thoughts on the Archaeological Thought", Cambridge Archaeological Journal, 3(2): 248-250.

- (1994): "Towards a cognitive archaeology". En C. Renfrew y E. Zubrow (eds.): The ancient mind. Elements of cognitive archaeology. New Directions in Archaeology.Cambridge University Press: 3-12.

RENFREW, C. y ZuBROw, E. (eds.) (1994): The ancient mind. Elements of cognitive archaeology. New Directions in Archaeology. Cambridge University Press. Cambridge.

Shanks, M. (1992): Experiencing the Past. On the character of archaeology. Routledge. London.

Shanks, M. y Tilley, Ch. (1987): Social Theory and Archaeology. Polity Press. Cambridge.

SCHWEDER, R.A. (1991): Thinking through cultures. Expeditions in Cultural Psychology. Harvard University Press. Cambridge, Massachusetts.

Stigler, J.W.; Shweder, R.A. and Herdt, G. (1990): $\mathrm{Cul}$ tural Psychology. Essays on comparative human development. Cambridge University Press. Cambridge. 